\title{
Évaluation du travail d'anglais de spécialité en semi-autonomie
}

\section{Christine Vaillant}

\section{OpenEdition}

Journals

Édition électronique

URL : http://journals.openedition.org/asp/4395

DOI : 10.4000/asp.4395

ISSN : 2108-6354

Éditeur

Groupe d'étude et de recherche en anglais de spécialité

Édition imprimée

Date de publication : 1 mars 1993

Pagination : 475-483

ISSN : 1246-8185

Référence électronique

Christine Vaillant, «Évaluation du travail d'anglais de spécialité en semi-autonomie », ASp [En ligne], 1 | 1993, mis en ligne le 03 juin 2004, consulté le 01 mai 2019. URL : http://journals.openedition.org/ asp/4395 ; DOI : 10.4000/asp.4395

Ce document a été généré automatiquement le 1 mai 2019.

Tous droits réservés 


\title{
Évaluation du travail d'anglais de spécialité en semi-autonomie
}

\author{
Christine Vaillant
}

1 L'objectif de cette communication est de poser le problème de l'évaluation dans des formations en semi-autonomie d'anglais de spécialité. L'expérience du projet pilote, d'enseignement en multimédia distance, du module d'anglais du DUT de Gestion servira d'illustration notre propos. Il s'agit donc bien d'inviter à une réflexion, à partir d'un exemple concret et non de faire un état de l'art de la docimologie.

2 L'expérience étudiée ici est celle d'un projet pilote lancé en juin 1989 par la Mission des équipements pédagogiques et des nouvelles technologies (M.E.P.E.N.T.E) de la Direction des Enseignements Supérieurs du Ministère de l'Éducation nationale. Il s'agit d'une préparation au Diplôme universitaire de Technologie de Gestion des Entreprises et des Administrations en multimédia par correspondance avec regroupements. « Un Groupe de pilotage » composé d'un représentant de chaque IUT a travaillé à la mise au point de règles de fonctionnement pédagogiques et administratives qui ont été discutées avec de nombreux enseignants puis soumises aux autorités (C.P.N, M.E.P.E.N.T.E, Assemblée des Directeurs d'IUT, Assemblée des Chefs de Départements GEA) qui les ont approuvées.

Aucun document ne donne officiellement les raisons pour lesquelles le Ministère a décidé - ou accepté de tenter - la mise en œuvre de ce projet. Il n'est sans doute pas trop hardi d'avancer que ces raisons sont fondamentalement d'ordre économique et politique. Ces données ne sont pas de notre ressort direct. Par contre, il nous revient de gérer les implications pédagogiques qui en découlent, dont celle du statut de l'évaluation.

4 Cette nouvelle formule a débuté en octobre 1991 dans les IUT de Clermont-Ferrand, Grenoble, Lille, Nantes et Toulouse. Elle est ouverte à des étudiants de formation initiale qui choisissent pour des motifs personnels de ne pas suivre un enseignement présentiel, et à des étudiants de formation continue. Tous ces auditeurs ont à leur disposition, dans toutes les matières du programme du matériel pédagogique sur divers types de médias (papier, informatique, vidéo, audio, etc.) qu'ils trouvent dans ce que l'on nomme des centres de ressources. Ils travaillent donc seuls la majeure partie du temps et peuvent entrer en contact avec leurs enseignants via divers moyens de communication mis, eux 
aussi à leur disposition: téléphone, fax, messagerie télématique et courrier. Ils se regroupent tous avec leurs enseignants, environ tous les quinze jours (selon les arrangements locaux) à l'IUT, au cours d'une journée de "regroupement " partagée entre les diverses matières en cours d'enseignement. Ces regroupements ne sont pas des cours à proprement parler. Ils permettent de répondre aux questions de fond ou de méthodologie, qu'elles soient suscitées par le parcours pédagogique «officiel» ou rencontrées par un individu lors d'un cheminement individuel. Ces séances permettent aussi de préparer le travail de la quinzaine à venir en distribuant de nouveaux matériaux ou en reformulant certains objectifs.

5 La préparation complète du DUT se fait sur trois ans avec un système d'UV sous-divisées elles-mêmes en modules capitalisables, à raison d'un module par année et par matière.

6 Les 3 modules d'anglais, qui reprennent le programme officiel, s'appuient sur l'utilisation de 4 médias : une méthode écrite du commerce, les cassettes audio de cette méthode, un didacticiel de révision grammaticale et des cassettes vidéo en vente dans le commerce. Un "parcours pédagogique" établit un cheminement entre tous ces médias qui doit permettre à l'auditeur d'atteindre à la fin des trois années le niveau théorique requis en anglais par le programme officiel.

7 Une approche traditionnelle de l'évaluation ne poserait aucun problème de mise en œuvre. Si l'on considère que le niveau d'anglais du baccalauréat est requis pour tout inscrit en IUT, que ce niveau est homogène, que les objectifs à atteindre sont « objectifs » «quantifiables » et précisément définis par le programme officiel ${ }^{1}$.

8 Alors des contenus précis peuvent être clairement définis et calibrés et l'on peut élaborer des tests quantitatifs pertinents pour définir si ces objectifs sont atteints par les apprenants ou non. Des outils de tous ordres ne manquent pas et l'enseignant d'anglais se trouve plutôt devant l'embarras du choix. Il ne nous paraît pas suffisant de nous contenter de ces méthodes.

9 En effet, depuis quelques années on assiste à une évolution pédagogique qui déplace la « source d'énergie » de l'enseignant, vers l'apprenant. Lors d'une communication au Congrès de l'APLIUT à La Rochelle en juin 1991, Hélène Canto de l'Université de Toronto, parlant de l'EAO a synthétisé cette évolution en ces termes :

[...] Une formation théorique à l'EAO reste encore à mettre en place. Car l'EAO bouleverse les données traditionnelles de l'enseignement et de l'apprentissage ; il individualise la discipline à enseigner, au centre du processus d'apprentissage : il place l'apprenant et non plus la discipline à enseigner, au centre du processus d'apprentissage ; il individualise la progression, respectant les rythmes de chacun ; il valorise l'utilisateur qui acquiert un sentiment de supériorité sur cette machine sophistiquée qu'il est capable de commander; il transforme l'évaluation en supprimant le "syndrome de la faute" : ce n'est plus l'enseignant qui sanctionne définitivement l'erreur, le programme permet à l'apprenant d'identifier l'origine de sa faute, de la corriger, et par conséquent de l'annuler. L'évaluation n'est alors plus une évaluation quantitative des résultats obtenus mais plutôt une analyse qualitative des processus d'apprentissage, du développement de nouvelles façons d'apprendre [...]. (1992: 55)

10 Le vieil adage britannique qui veut que pour enseigner le latin à John, il faille connaître John est à l'ordre du jour. Ces constatations ont été déjà faites dans le passé par des auteurs tels que H. Holec ou J.-P. Narcy par exemple. Centrée traditionnellement sur l'enseignant face aux apprenants, la pratique pédagogique évolue vers la valorisation de la relation apprenant/objet d'étude, l'enseignant reprenant son rôle de guide, conseiller 
et maître. Viennent s'ajouter l'attention portée au choix des supports utilisés et aux stratégies d'apprentissage des individus. Le nombre des paramètres augmente donc permettant une plus grande individualisation des objectifs, des stratégies pédagogiques et du suivi des apprenants. L'apprentissage de l'anglais de spécialité dans des formations universitaires, conjugué à une mise à disposition d'outils et de supports pédagogiques variés favorise particulièrement cette évolution.

11 En effet, si l'on reprend l'exemple du DUT de Gestion que voit-on? Le niveau initial en langue est extrêmement hétérogène. Les étudiants qui viennent d'obtenir leur baccalauréat, outre les disparités personnelles de niveau, arrivent de sections différentes (B, G2, C et quelques A).

12 Les étudiants de formation continue, plus âgés présentent des profils encore plus diversifiés. Le programme officiel, par ailleurs, ambitieux dans ses termes, laisse en fait toute latitude d'interprétation et permet aux enseignants d'orienter leur enseignement dans de multiples directions et surtout de fixer des objectifs de niveau de compétence très éloignés les uns des autres. L'enseignement d'anglais de spécialité à des étudiants de matières économiques et de gestion doit à la fois s'appuyer sur un substrat déjà appris et réputé acquis pour construire une performance linguistique indispensable dans le monde des affaires et le monde moderne en général, tout en sachant que (pour des raisons complexes qui ne remettent pas en cause le travail des enseignants du secondaire) ce substrat est pour le moins défaillant.

13 Nous avons donc, dans le cas du DUT de gestion en multimédia avec regroupement pris le parti de privilégier la «qualité » de l'évolution des performances sur l'acquisition d'une "quantité » de connaissances ou de performances communes à tous (nonobstant le niveau du substrat ou le vécu de l'individu).

14 Chaque auditeur doit commencer par faire le point sur son propre passé linguistique. Nous l'invitons ensuite à réfléchir à ses stratégies pédagogiques et à fixer, avec l'enseignant, en fonction de tous ces éléments, ses objectifs pédagogiques. Ces procédures ont été depuis longtemps étudiées et mises en pratique au C.R.A.P.E.L de Nancy. La spécificité de notre problématique est sans doute que nous devons concilier cette individualisation des parcours personnels avec l'objectif commun du programme officiel.

On se retrouve donc dans une situation où, très rapidement, la diversité des tâches, les disparités de rythme et de parcours pédagogiques ne sont plus masquées par la «normalisation » imposée par des cours plus traditionnels. Se pose alors avec acuité la question de l'évaluation de ce nouveau type « d'apprentissage ». Il ne serait pas honnête de revenir à une évaluation normative collective du type traditionnel après avoir entrainé l'apprenant vers un apprentissage personnalisé et individualisé, qui de plus, l'expérience le prouve, permet dans beaucoup de cas de dépasser des blocages profonds vis-à-vis de l'anglais. Le choix d'un enseignement en semi-autonomie a été, en partie, dicté par les impératifs de "multimédiatisation » et de délocalisation imposés par le Ministère, mais il correspond plus profondément à un parti pris pédagogique de l'équipe de pilotage. Philippe Carre (1990) exprime parfaitement ce point :

La finalité de l'auto-formation .assistée n'est en effet pas seulement de permettre une plus grande souplesse d'organisation quant aux rythmes, lieux, durées et modalités individuelles d'apprentissage, ni avant de favoriser des économies d'échelle, bien que ces objectifs doivent être atteints du même coup. L'autoformation dans l'apprentissage des langues permet avant tout une meilleure efficacité de la formation, en développant chez l'apprenant une attitude de 
responsabilisation, donc de production de son savoir, en lieu et place des attitudes de consommation passive qui caractérisent trop souvent les approches traditionnelles centrées sur l'enseignant ou le programme.

16 Dans la logique de ce qui vient d'être énoncé, nous nous retrouvons dans une situation paradoxale où, si nous imposons à tous l'objectif « maximal » du programme officiel nous fixons ipso facto un niveau "élevé » d'évaluation, mais nous réduisons par le même mouvement la diversité féconde des objectifs personnalisés.

Nous avions donc à concevoir un outil d'évaluation qui permette de prendre en compte tous ces éléments. Les auditeurs ont été invités, en fin de module d'enseignement et dans des conditions d'examen, à faire le point sur leurs acquis et sur leurs manques. À l'aide d'un questionnaire-guide, ils devaient rappeler les objectifs qu'ils s'étaient fixés en début d'apprentissage - et que nous avions gardés - et indiquer les points de réussite et d'échec. Ils devaient indiquer les outils qu'ils avaient le plus utilisés et expliquer pourquoi ils avaient négligé ou rejeté les autres. Invités constamment lors du module à utiliser un maximum de sources «extérieures" à celles que nous avions proposées, ils devaient indiquer celles qu'ils avaient exploitées et en donner une description et une appréciation argumentée. Le dernier volet leur demandait de relater en anglais et en une centaine de mots leur expérience générale du multimédia. Enfin ils devaient s'attribuer une note tenant compte de la quantité et de la qualité du travail qu'ils avaient fourni en anglais. Ce dossier a ensuite été noté par l'enseignant. De la moyenne des deux a résulté la note finale. On a pu constater que les apprenants s'étaient globalement jugés avec, sinon sévérité, du moins beaucoup de justesse. Les notes que nous avons attribuées ont très généralement confirmé ou relevé celles des étudiants. Les questions du questionnaire semblent pertinentes et la lecture des réponses permet une vision «qualitative » du travail fourni tout à faire comparable à la vision « quantitative » de copies traditionnelles.

Cette manière de procéder, très pragmatique, apparaît (sous réserve de confirmation sur un plus large échantillon dans les années qui viennent) tout à fait adaptée à la démarche du module de $1^{\text {re }}$ année.

Nous travaillons actuellement sur l'évaluation du module de $2^{\mathrm{e}}$ année dont nous sommes en train d'achever la conception du parcours pédagogique. Le problème de l'évaluation de l'apprentissage de l'anglais de spécialité en semi-autonomie apparaît particulièrement clairement dans cet exemple d'un enseignement sanctionné par un diplôme national, mais pour être moins urgent, il n'est pas moins présent dans des formations non diplômantes, en formation continue par exemple. Il ne s'agit donc pas pour nous de faire partager à d'autres collègues un problème que nous aurions seuls à résoudre. Il s'agit bien de réfléchir d'une manière plus générale aux implications didactiques et pédagogiques de nouveaux types d'apprentissage et aux manières de mettre en place, de nouvelles procédures d'évaluation formative, sommative ou fonctionnelle. 


\section{BIBLIOGRAPHIE}

Bireaud, Annie. 1990. « Pédagogie et méthodes pédagogiques dans l'enseignement supérieur ».

Revue française de pédagogie 91, 13-24.

Caillet, E. et E. Barchechat. 1990. Sur la formation des formateurs au multimédia Séminaire de recherche-développement DFP, CNAM, EUROTECNET.

Canto, H. 1992. « Un didacticiel pour l'enseignement de l'intonation en français langue étrangère (FLE) ». Les Cahiers de l'APLIUT 11/3, 51-59.

Carre, Ph. 1990. Organiser l'apprentissage des langues étrangères. Paris : Les Éditions d'Organisation. Changeux, J.-P. 1983. L'homme neuronal. Paris : Fayard.

Chapolthier, G. 1988. Mémoire et cerveau. Biologie de l'apprentissage. Monaco : Éditions du Rocher.

Duda, R., H. Moulden et M. Rees. 1988. « Assessing metacognitive ability ». Mélanges pédagogiques, Université Nancy 2, 59.

Eco, U. 1985. La guerre du faux. Paris : Grasset et Fasquelle.

Ellis, G et B. Sinclair. 1989. Learning to Learn English. Cambridge : Cambridge University Press. Garanderie de la, A. 1982. Pédagogie des moyens d'apprendre. Paris : Centurion.

Habermas, J. 1987. Morale et communication. Paris : Cerf.

Hagège, C. 1986. L'Homme de paroles. Paris : Fayard.

Hatch, E. 1983. Psycholinguistics. Rowley, MA : Newbury House.

Henri, F. et A. Kaye. 1985. Le savoir à domicile. Pédagogie et problématique de la formation à distance. Sainte-Foy : Presses de l'Université du Québec, Télé-Université.

Holec, H. 1988. Autonomie et apprentissage auto-dirigé : terrains d'application actuels. Strasbourg : Conseil de l'Europe.

Narcy, J.-P. 1990. Apprendre une langue étrangère, Didactique des langues. Paris : Les Éditions d'Organisation.

Nora, S. et A. Minc. 1978. L'informatisation de la société. Paris : La Documentation Française.

Phillips, M. 1987. Communicative Language Learning and the Microcomputer. Londres : The British Council.

Piaget, J. P. 1970. Psychologie et épistémologie, pour une théorie de la connaissance. Paris : Gonthier Denoël.

Sabah, G. 1988. L'Intelligence artificielle et le langage. Paris : Hermès.

Trocme-Fabre, H. 1987. J'apprends, donc je suis. Paris : Les Éditions d'Organisation.

\section{NOTES}

1. Programme officiel DUT GEA Arrêté du 9 août 1988 B.O n 44 du 22 décembre 1988 


\section{AUTEUR}

\section{CHRISTINE VAILLANT}

IUT A Département GEA 1 Université Toulouse 3. christine.vaillant@free.fr 